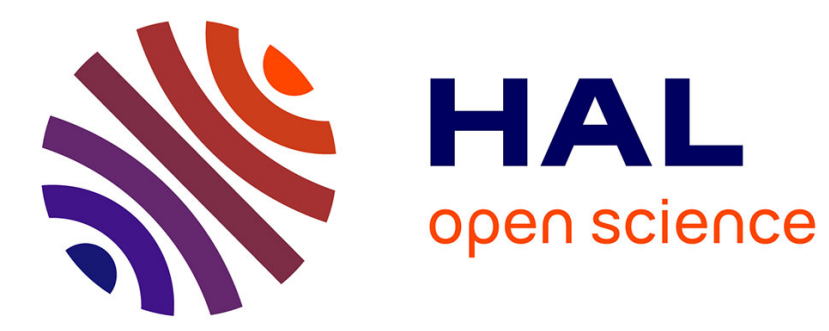

\title{
Exploring the Atwood-number dependence of the highly nonlinear Rayleigh-Taylor instability regime in high-energy-density conditions
}

G. Rigon, B. Albertazzi, P. Mabey, Th Michel, E. Falize, V. Bouffetier, L.

Ceurvorst, L. Masse, M. Koenig, A. Casner

\section{To cite this version:}

G. Rigon, B. Albertazzi, P. Mabey, Th Michel, E. Falize, et al.. Exploring the Atwood-number dependence of the highly nonlinear Rayleigh-Taylor instability regime in high-energy-density conditions. Physical Review E , 2021, 104 (4), 10.1103/PhysRevE.104.045213 . hal-03406699

\section{HAL Id: hal-03406699 \\ https://hal.science/hal-03406699}

Submitted on 28 Oct 2021

HAL is a multi-disciplinary open access archive for the deposit and dissemination of scientific research documents, whether they are published or not. The documents may come from teaching and research institutions in France or abroad, or from public or private research centers.
L'archive ouverte pluridisciplinaire HAL, est destinée au dépôt et à la diffusion de documents scientifiques de niveau recherche, publiés ou non, émanant des établissements d'enseignement et de recherche français ou étrangers, des laboratoires publics ou privés. 


\title{
Exploring the Atwood-number dependency of the highly nonlinear Rayleigh-Taylor Instability regime in High Energy Density conditions
}

\author{
G. Rigon, ${ }^{1,2, *}$ B. Albertazzi, ${ }^{1}$ P. Mabey,${ }^{1,3}$ Th. Michel,,${ }^{1}$ E. Falize,${ }^{4}$ \\ V. Bouffetier, ${ }^{5}$ L. Ceurvorst, ${ }^{5}$ L. Masse, ${ }^{4}$ M. Koenig, ${ }^{1,6}$ and A. Casner ${ }^{7,5}$ \\ ${ }^{1}$ LULI, CNRS, CEA, Ecole Polytechnique, UPMC, Univ Paris 06: Sorbonne Universites, \\ Institut Polytechnique de Paris, F-91128 Palaiseau cedex, France \\ ${ }^{2}$ JSPS International Research Fellow, Graduate School of Science, \\ Nagoya University, Furo-cho, Chikusa-ku, Nagoya 464-8602, Japan \\ ${ }^{3}$ Freie Universität Berlin, Department of Physics, Arnimallee 14, 14195 Berlin, Germany \\ ${ }^{4}$ CEA-DAM, DIF, F-91297 Arpajon, France \\ ${ }^{5}$ Université de Bordeaux-CNRS-CEA, CELIA, UMR 510\%, F-33405 Talence, France \\ ${ }^{6}$ Graduate School of Engineering, Osaka University, Osaka, 565-0871, Japan \\ ${ }^{7}$ CEA-CESTA, 15 avenue des Sablières, CS 60001, 33116 Le Barp Cedex, France
}

\begin{abstract}
We experimentally study the late time, highly nonlinear regime of the Rayleigh-Taylor Instability in a decelerating phase. A series of laser-driven experiments is performed on the LULI2000 laser, in which the initial Atwood number is varied by adjusting the decelerating medium density. The high power laser is used in a direct drive configuration to put into motion a solid target. Its rear side, which initially possesses a two-dimensional machined sinusoidal perturbations, expands and decelerates into a foam leading to a Rayleigh-Taylor unstable situation. The interface position and morphology are measured by time-resolved x-ray radiography. We develop a simple Atwood dependent model describing the motion of the decelerating interface, from which its acceleration history is obtained. The measured amplitude of the instability, or mixing zone width, is then compared with late time acceleration dependent Rayleigh-Taylor instability models. The shortcoming of this classical models, when applied to high energy density conditions, is shown. This calls into question their uses for systems, where a shock wave is present, such as those found in laboratory astrophysics or in Inertial Confinement Fusion.
\end{abstract}

The article is published in Physical Review E 104, 045213 (2021):

https://journals.aps.org/pre/abstract/10.1103/PhysRevE.104.045213

DOI: https://doi.org/10.1103/PhysRevE.104.045213

This is an author format version (preprint), in compliance with American Physical Society Transfer of Copyright Agreement.

Copyright 2021 American Physical Society.

\section{INTRODUCTION}

Rayleigh-Taylor instability (RTI) [1, 2] is a seminal hydrodynamic instability, ubiquitous in the Universe, which pervades at all scales from Bose-Einstein Condensates [3] to astrophysical distances $[4,5]$. This instability leads to the growth of the perturbation at the interface between fluids as soon as the scalar product between pressure and density gradients is negative. On Earth, this necessary condition is reached when a denser fluid rests on top of one of lower density, or in case of interface acceleration. RTI has therefore a great impact on fluids dynamics in numerous systems [6-11].

Despite its apparent simplicity, RTI remains nowadays an active field of study as testified by the recent reviews [12-14]. This is partially due to the complexity of all non-linear systems in fluid dynamics. Indeed, strikingly, even the late-time growth of single mode RTI is not well

\footnotetext{
* gabriel.rigon@polytechnique.edu
}

understood [15]. After the linear phase of growth, the instability pattern becomes asymmetric [16]. The heavy fluid falls as spikes into the lighter fluid and the lighter fluid rises as bubbles into the heavy fluid due to buoyancy forces. A key parameter is the initial Atwood number $A_{t}=\left(\rho_{1}-\rho_{2}\right) /\left(\rho_{1}+\rho_{2}\right), \rho_{i}$ being the $i$-fluid density. Based on the potential flow theory of Layzer [17], a terminal bubble velocity was proposed by Goncharov for any Atwood number [18]. However, this theory breaks down for 3D single mode as numerically evidenced for various Atwood number [19, 20]. A re-acceleration stage is predicted to occur for the bubble velocity when the secondary Kelvin-Helmholtz instabilities develop along the RTI spikes. Vorticity accumulates inside the RTI bubble inducing its re-acceleration [21]. A similar phenomenon was demonstrated at the ablation front in the context of Inertial Confinement Fusion (ICF) [22]. RTI in the acceleration $[10,11]$, or deceleration phase $[23-25]$, remains a major challenge towards a burning plasma in ICF. From an experimental point of view, while RTI has been widely studied in shock tubes experiments [26], a parametric scan in Atwood requests different gas mix- 


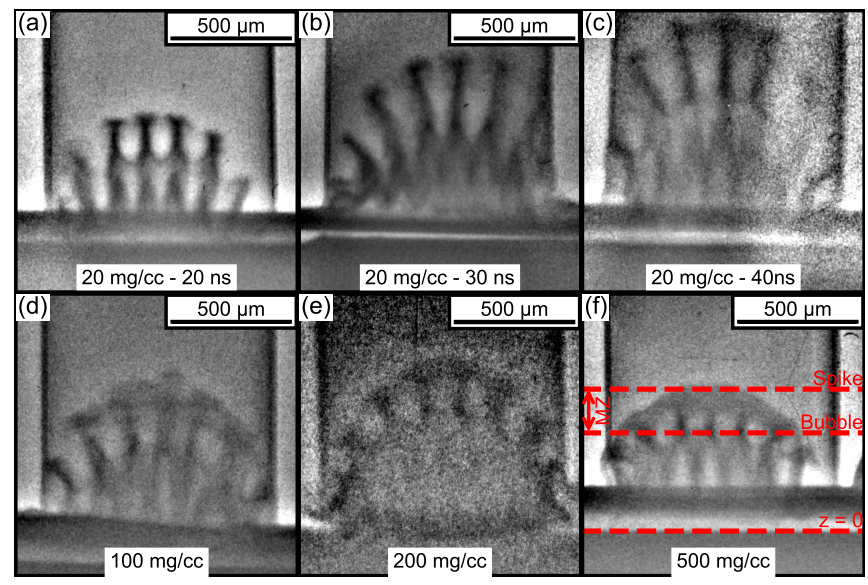

FIG. 1. Example of obtained radiographies. The experimental radiographies (a), (b), (c) show the dynamic of the evolution of a $20 \mathrm{mg} \mathrm{cm}^{-3}$ foam. Similarly (d), (e), (f), corresponds to radiographs of $(100,200$ and 500$) \mathrm{mg} \mathrm{cm}^{-3}$ foams taken $30 \mathrm{~ns}$ after laser shot. The initial wavelength, $\lambda$, is $120 \mu \mathrm{m}$.

tures [27, 28]. Furthermore, a precise control and knowledge of the initial conditions remains challenging for any RTI experiments [29, 30]. High Energy Density (HED) settings allow to circumvent these intrinsic limitations $[31,32]$.

In this article, we explore the Atwood-number dependency of the RTI in HED conditions for the first time. This study builds upon the classical scheme of a laserproduced plasma expanding into a foam [33-36], with different densities. This HED platform is much simpler than traditional shock tube RTI platforms. Thanks to this simplicity, data sets are obtained and a parametric study, exploring different initial conditions, is performed. Accurate measurements of acceleration and mixing zone widths are made and compared to classical models which failed to reproduce the measurements. Explanations are suggested to understand the higher than expected RTI growths, a trend already observed in previous HED RTI settings [37, 38].

\section{EXPERIMENTAL SETUP}

The experiments were performed on the LULI2000 laser facility using the setup described in [35]. The nano2000 laser beam $(500 \mathrm{~J}, 2 \omega, 1.5 \mathrm{~ns}$ with a $470 \mu \mathrm{m}$ super-Gaussian focal spot) deposits its energy on a multilayer target, with an intensity of $2 \times 10^{14} \mathrm{~W} \mathrm{~cm}^{-2}$. The target is composed of a $10 \mu \mathrm{m}$ parylene ablator, a $1 \mu \mathrm{m}$ gold x-ray shielding layer, and a $40 \mu \mathrm{m}$ modulated pusher $\left(\mathrm{C}_{8} \mathrm{H}_{7} \mathrm{Br}\right)$. We employed three kind of modulations: a sine curve $(\lambda=120 \mu \mathrm{m}$ wavelength, $10 \mu \mathrm{m}$ amplitude), the sum of two sine curves ((70 and 130) $\mathrm{um}$ wavelengths, (10 and 10) $\mu \mathrm{m}$ amplitudes), and flat targets. Here we will focus on single-mode data, as no obvious difference was observed between mono- and bi-mode [35]. As a consequence of the target ablation by the laser, a shockwave is launched into the target and put it into motion. The pusher expands into an external medium, a resorcinol formaldehyde $\left(\mathrm{C}_{15} \mathrm{H}_{12} \mathrm{O}_{4}\right)$ foam. Four foam densities were used: $\left(20,100,200\right.$ and 500) $\mathrm{mg} \mathrm{cm}^{-3}$, leading to an initial Atwood number of 0.97, 0.87, 0.75 and 0.47 respectively. Since the ablation pressure applied by the laser is maintained only for $\sim 1.5 \mathrm{~ns}$ and since the foam has a non-negligible density, the expanding pusher decelerates resulting in a RT unstable situation.

To diagnose the interface between pusher and foam $\mathrm{x}$-ray radiographs were taken (see Fig. 1). Those radiographs were performed by point projection [39], with an $\mathrm{x}$-ray source produced by the pico2000 laser $(55 \mathrm{~J}, 10 \mathrm{ps})$ focused on a $25 \mu \mathrm{m}$ titanium wire (K $\alpha$ x-ray emission line: $\sim 4.5 \mathrm{keV}$ ) and an imaging plate detector. This diagnostic presents a static $\lesssim 25 \mu \mathrm{m}$ resolution [35]. It allows us to distinguish between pusher and foam, the contrast between the two being enhanced by the difference in density as well as the bromine doping of the pusher $(\sim 40 \%$ in mass). From those radiographs, two parameters are measured: the position of the central RTI spike, and position of the central bubble extremities (cf. Fig. 1 (f)). We call the distance between spike and bubble extremities, mixing zone (MZ) width. In the case of the flat targets, the only observed variable is the actual interface position, which is needed to determine the RTI growth. To obtain the overall dynamics, we combine different single snapshot radiographs acquired from similar targets, laser drive and initial conditions (see Fig. 1 (a,b,c)). To compensate the laser energy fluctuations, we normalized the time delays with respect to the laser energy as explained in $[35,40]: \tilde{t}=t\left(E / E_{0}\right)^{1 / 3}$. Here we take $500 \mathrm{~J}$ as the reference laser drive energy $\left(E_{0}\right)$.

To build more confidence in the experiments, we have conducted a series of radiation-hydrodynamics simulations using FLASH (v.4.5) [41, 42]. We used it in a 2D Cartesian geometry in a hydrodynamic setup with three temperatures and an adaptive mesh refinement (paramesh4dev). We employed an "hllc" Riemann solver, an "mc" slope limiter, with a second order data reconstruction (type MUSCL-Hancock) and a cfl of 0.3 in an unsplit solver scheme. The simulation domain, we employed, is a square with a length, which varies between (800 and 1400) $\mu \mathrm{m}$ depending on the simulated case (far reaching or not). It is divided in four main blocks, each subject to adaptive mesh refinement (AMR) up to the sixth order, and further divided in 16 cells in each spatial direction. This leads to a maximal resolution varying between (0.8 and 1.4) $\mu \mathrm{m}$ depending on the domain size. The AMR is based on the variation of density, temperature (electronic and ionic) and pressure. The laser intensity $\left(\simeq 5 \times 10^{13} \mathrm{~W} \mathrm{~cm}^{-2}\right)$ was adjusted to reproduce the initial experimental velocity for the interface between pusher and foam. And the laser pulse was model using 50 temporally equidistant points spanning from 0 to 2 ns. All the simulated plastic layers use the same IONMIX [43] tabulated equation of state (polystyrene) as 
well as their respective tabulated opacity calculated with PROPACEOS [44] with 40 radiations groups. The different elements of the experiment except the gold layer, which is too thin, are initialized in accordance with the geometry of the experiment (vacuum, ablator, pusher, foam and tube).

In addition to these simulations, 1D simulations in Cartesian geometry were performed for the need of the alteration of buoyancy-drag model discuss later. These simulations employed the same parameters as the $2 \mathrm{D}$ simulations except for the initial geometry and the laser intensity, which need to be lowered to obtain the right initial velocity.

\section{INTERFACE MOTION}

\section{A. Measure of the position}

We focus first on the motion of the interface between pusher and foam. Here by interface, we mean the position where the interface would be in the absence of RTI effects. Indeed, this motion triggers the consequent RTI growth. Since the interface is warped, due to the development of hydrodynamic instabilities, we theoretically can't follow its motion except for the flat targets. As shown on Fig. 2 (a), the movement of the interface is equal to the movement of the central RTI spike of the modulated targets. The difference in position is too small to distinguish between the two with our experimental resolution. This is also observed in simulations. On Fig. 2 (b), the length of the spikes and bubbles is displayed. Here, this length is calculated as the difference between their respective position and the position of the interface obtained through simulation $\left(x_{\text {spike }}-x_{\text {interface }}\right.$ and $\left.x_{\text {interface }}-x_{\text {bubble }}\right)$. As can be seen, the spike length is lower than $100 \mu \mathrm{m}$ (except for two out-of-curve experimental points), and has a minimal error of $25 \mu \mathrm{m}$, which is the ideal static case. Thus, the experimental position of the spike and of the interface can be hardly distinguished experimentally. This is especially the case considering the experimental "length of the interface" (black dots), which should be equal to zero but is of the order of the length of the spikes. This is mainly due to a defect in the spike growth resulting from the proximity of the shock wave [45]. This fact is obvious in simulation as shown on Fig.2 (c), where the pressure and the interface morphology are displayed. The RTI spikes are subject to an excessive pressure, which reduces their growth. Thus, the position of the spikes corresponds approximately to the interface one. As for the bubbles, they are easily distinguished from the spikes and interface.

The experimental data displayed on Fig. 3 (a) thus correspond to the interface dynamics for each foam density. Each dynamics can be divided in two phases: ballistic and decelerating. The first phase corresponds to a nearly constant interface velocity. If we compare our experiment to the classical model of Supernovae Remnants

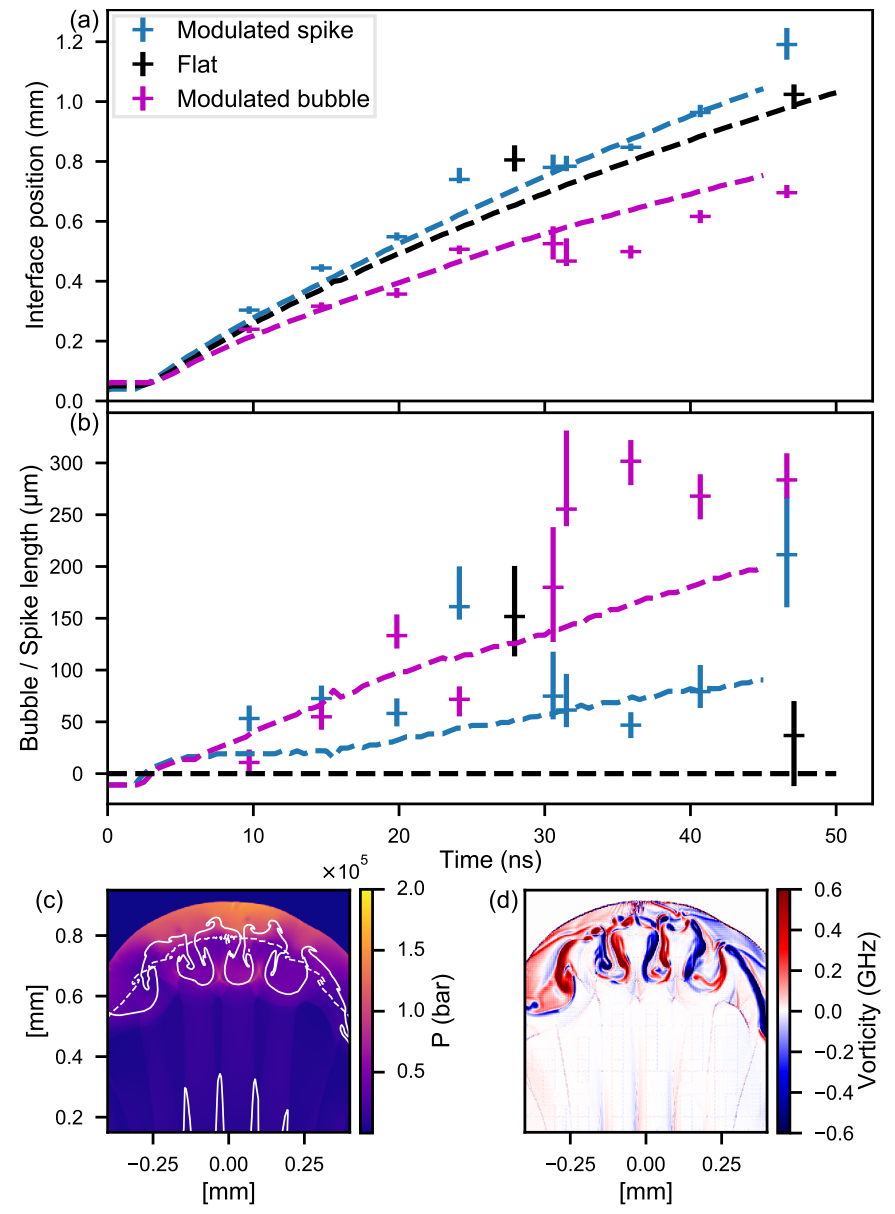

FIG. 2. Comparison between RTI spike and interface position. (a) Temporal evolution of the position of the spike (blue), bubble (magenta) and interface of a flat target (black) both experimentally (points data) and in simulation for a $20 \mathrm{mg} \mathrm{cm}^{-3}$ foam. (b) Temporal evolution of the bubble and spike lengths, which are calculated using the simulated interface position from (a). (c) Simulated pressure map taken $25 \mathrm{~ns}$ after shock break out around the interface. The interface between pusher and foam is depicted with a white full line for the modulated case and a dashed line for a flat target. (d) Map of the simulated vorticity in the $z$ direction (curl of velocity projected on $z$ axis) in the modulated case.

(SNR) expansion [46, 47], this phase would correspond to the free expansion where the mass of swept external medium is still too small to influence the shock and interface dynamics. Here, the interface velocity does not depend on the foam density, but on the ablator composition and on the momentum transmitted by the laser, both parameters kept constant. Following the SNR analogy, this ballistic phase ends as soon as a sufficient mass of foam is swept by the shock, which obviously happens sooner with higher foam density. In the following phase, the interface decelerates triggering the RTI growth. As expected, the deceleration increases with higher foam density. This is especially obvious with the two extreme cases ((20 and $\left.500) \mathrm{mg} \mathrm{cm}^{-3}\right)$ : for the low density case, the motion re- 


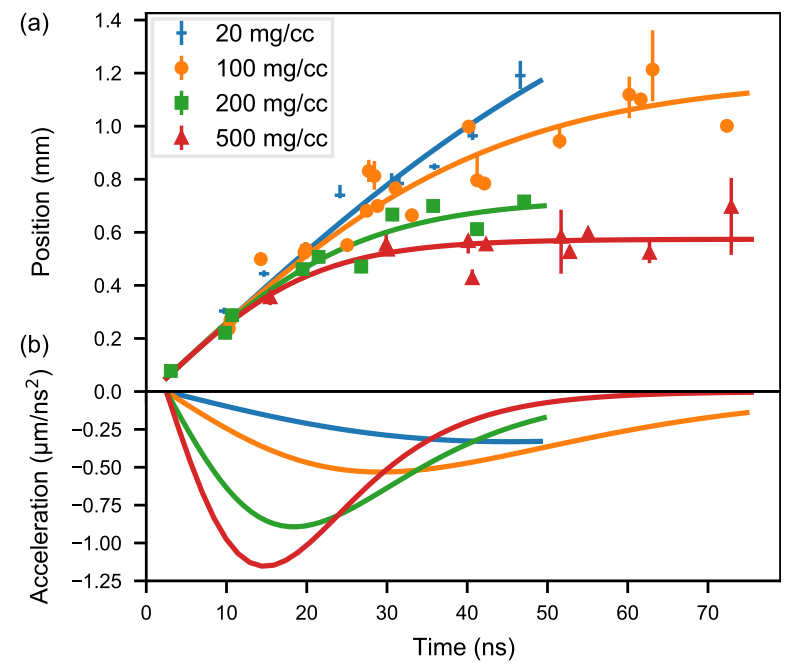

FIG. 3. Temporal dynamics of the interface. (a) Evolution of the interface position for the different foam densities (20, 100, 200 and 500) $\mathrm{mg} \mathrm{cm}^{-3}$ corresponding to $A_{t}=0.97,0.87$, 0.75 and 0.47 . The scattered data points correspond to the obtained experimental results. The associated curves correspond to the result of our model (1) with an initial velocity fixed to $28 \mathrm{um} \mathrm{ns}^{-1}$. (b) Acceleration of the interface deduced from the model $(\ddot{x}(t))$.

mains ballistic, whereas it becomes quasi-stationary for the high one. Varying the initial foam density induces two opposing effects expected from the linear approximation of the RTI growth: a high density leads to a high deceleration, thus a high growth rate $(\propto \sqrt{g})$, and a low Atwood number, thus a low growth rate $\left(\propto \sqrt{A_{t}}\right)$.

\section{B. Analytical model}

Before studying in detail the RTI growth, we develop an analytical approach of the interface motion. The following 1D analysis is based on two hypothesis: (i) the initial velocity of the interface, $v_{0}$, is the same for every foam density, (ii) the deceleration of the interface depends on the swept mass of foam and on the interface velocity. The first assumption is a given since the initial velocity of the interface depends on the momentum transmitted by the shock wave, which is the same for every targets (same target composition and laser intensity). The dependence of the acceleration on the swept mass is not a far stretch in 1D. Indeed, the shocked foam accumulates at the pusher front leading to an increased mass thus inertia. If we suppose the deceleration being proportional to the swept mass then it will be proportional to the shock (or interface) position $\left(\ddot{x} \propto m_{\text {swept }} \propto x \rho_{0}\right)$. Finally, the dependence of the deceleration on the interface velocity can be understood when considering that in a time step the pusher has to sweep a foam quantity proportional to its velocity $(\ddot{x} \propto \dot{x})$. Therefore, the interface dynamics should follow the differential equation:
$\ddot{x}=-B x \dot{x}$, with $B \propto \rho_{0}$ a constant. This equation admits for solution:

$$
x(t)=\sqrt{\frac{2 v_{0}}{B}} \tanh \sqrt{\frac{B v_{0}}{2}} t
$$

Here, $t=0$ corresponds to the shock break out (2.5 ns) and $x=0$ to the position of the interface at that time $(50 \mu \mathrm{m})$. This coordinate system is a space-time translation of the experimental coordinates. It is used only in the following paragraph. We can fit this solution to our experimental results as shown on Fig. 3 (a). If all fitting parameters are unconstrained then the initial velocity, $v_{0}=\dot{x}(t=0)$, varies between (25.9 and 29.3) $\mu \mathrm{mns}^{-1}$ depending on the data set. Since this variation is small and since all the initial velocities should be the same, we impose a $v_{0}$ of $28 \mu \mathrm{mns}^{-1}$, which corresponds to our simulated data. $B$ is then equal to $(1.7 \pm 0.2,4.3 \pm 0.1$,

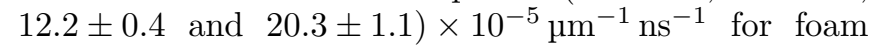
density of (20, 100, 200 and 500) $\mathrm{mg} \mathrm{cm}^{-3}$ respectively. As predicted we find that $B$ is nearly proportional to the foam density $\left(B / \rho_{0}=(4.3 \pm 1.0) \times 10^{5} \mu \mathrm{m}^{2} \mathrm{mg}^{-1} \mathrm{~ns}^{-1}\right)$. The observed variation might be linked to some inaccuracy on the foam density or on our experiments process. Given our model, we can calculate the acceleration of the interface as a function of time (see Fig. 3 (b)). The temporal position of the maximum of each deceleration can be calculated $\left(\log (2+\sqrt{3}) / \sqrt{2 B v_{0}}\right)$, thus determining the transition between the two phases (ballistic and decelerating). Here the maximal deceleration is attained at (43, 27, 16 and 12) ns after shot break-through (2.5 ns) for each respective density. The model was also tested on experimental results obtained on SACLA (Japanese XFEL) for the same kind of target $\left(100 \mathrm{mg} \mathrm{cm}^{-3}\right)$ but with a lower laser intensity, $\sim 10^{13} \mathrm{~W} \mathrm{~cm}^{-2}$ [36]. Despite the lower initial interface velocity $v 0=(10.5 \pm 0.2) \mathrm{\mu m} \mathrm{ns}^{-1}$, the model fit the data with the same $B$ parameter as in LULI2000.

We should mention that the deceleration exists from the beginning of the interface motion according to our model (see Fig. 2 c). In that sense, the first phase of the evolution would be closer to the ejecta dominated self-similar (we do not know if there is self-similarity in our experiment) phase of the SNR dynamic [48, 49]. To complete this parallel, we should add that the subsequent Sedov-Taylor phase should start when the mass of swept up foam equal the expanding pusher mass. Assuming that our problem is mono-dimensional (no lateral expansion) and that the whole depth of the pusher is put in motion, then the phase will start (4.1, 10.6 and 21.9) ns after shock break-out for the (500, 200 and 100) $\mathrm{mg} \mathrm{cm}^{-3}$ foam respectively. In the case of the $20 \mathrm{mg} \mathrm{cm}^{-3}$, the mass swept up in the foam is never equivalent to the mass of the pusher, the later being too dense. This assumes, however, that our model stay true even at long time for this foam density. 


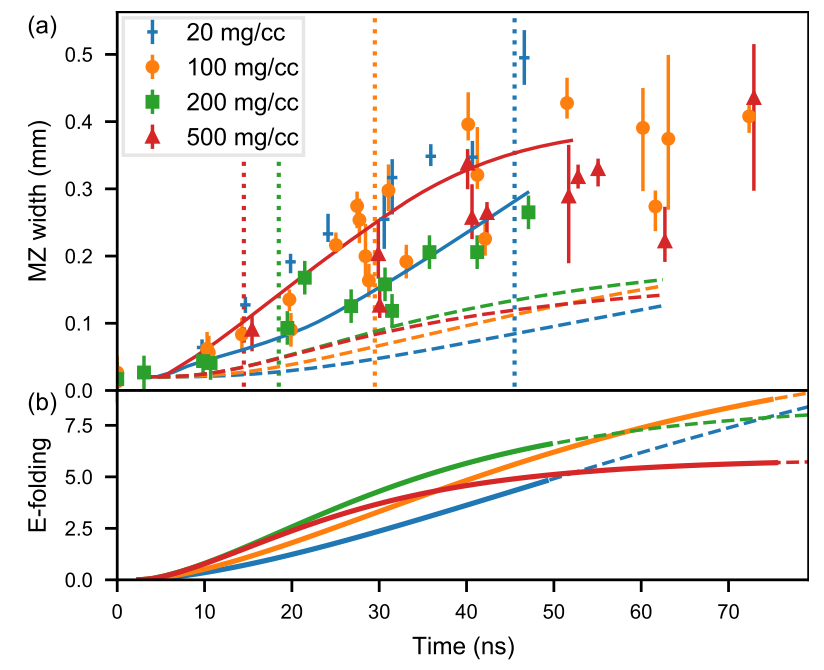

FIG. 4. (a) Evolution of the mixing zone (MZ) width as a function of time for different foam density: (20, 100, 200 and 500) $\mathrm{mg} \mathrm{cm}^{-3}$ corresponding to $A_{t}=0.97,0.87,0.75$ and 0.47. The vertical dotted lines indicate the time of maximum deceleration for each density, at (45.5, 29.5, 18.5 and 14.5) ns respectively. The dashed lines correspond to a modified buoyancy-drag model [50] using the experimental parameters (initial Atwood number and modulation) and the deceleration from our model. The full lines correspond to the addition of $1 \mathrm{D}$ expansion to the previous model [51, 52]. (b) Number of e-foldings (with an extrapolation dashed) for each foam.

\section{GROWTH OF THE RTI}

We now consider the actual RTI growth, and measured MZ width dynamics. As clearly shown by the Fig. 4 (a), the growth of the MZ (which here corresponds approximately to the growth of the bubble) is faster with a high Atwood number. On Fig. 4 (a), we draw in vertical dotted lines the deceleration time for each foam density as calculated previously with our model. As seen on Fig. 3 (b), the deceleration attains its maximum at this time so should the RTI growth rate. When comparing the different RT growths, we can clearly see that the instability grows faster with the lower density foam $\left(20 \mathrm{mg} \mathrm{cm}^{-3}\right)$ than with the higher one $\left(500 \mathrm{mg} \mathrm{cm}^{-3}\right)$. This shows the preponderance of the effect of the foam density over the Atwood number compared to the deceleration. Two main explanations could be envisioned: either the difference in deceleration is too small to compensate for the difference in Atwood number, or non-linear effects affect the growth of the instability.

Let us consider the first hypothesis. If we take into account only the extreme foam values, (20 and $500) \mathrm{mg} \mathrm{cm}^{-3}$, their maximal decelerations are respectively $(0.33 \pm 0.02$ and $1.15 \pm 0.03) \mathrm{um} \mathrm{ns}^{-1}$, according to our model. Now if we consider their respective Atwood number unchanged (same compression and relaxation of the pusher and foam) then the growth rate of the higher density foam should be higher $\left(A_{t} g_{\max } \sim 0.54 \mu \mathrm{mns}{ }^{-2}\right.$ versus 0.32 for the $20 \mathrm{mg} \mathrm{cm}^{-3}$ foam). This is obviously not the case, even for early times (their deceleration cross only after $\sim 35 \mathrm{~ns}$ ). Thus the first hypothesis does not hold.

The second hypothesis is much more likely as the deformation of the interface is already well developed after a few nanoseconds, with an amplitude exceeding $0.1 \lambda$, the usual criteria for the appearance of non-linear effects [13]. As a result of non-linear effects, the RTI growth should become linear in time (more consistent with our late time results) and the bubble velocity should tend to an asymptotic value $[17,18]$ proportional to $\left(\left(\rho_{1}-\rho_{2}\right) g\right)^{0.5}$. The difference in density is obviously lower for higher foam density and the deceleration, which is initially higher, is quicker to tend towards zero. Since the deceleration is not sustained for high density foam, the RTI can not quite develop itself.

This asymptotic behavior in the RTI growth encounters a limit in the form of a re-acceleration [53]. Such phenomenon should theoretically happen when the amplitude of the instability reaches the value of its wavelength and the number of e-folding is around 8 (the efolding is defined as the integral of the growth rate $n(t)$ as a function of time) $[19,21]$. As shown in Fig. 4 (b), this value is not yet reached. However, those conditions should be reachable with our experimental design after 70 to $80 \mathrm{~ns}$ for the lighter foams (with the $500 \mathrm{mg} \mathrm{cm}^{-3}$ foam it will never be reached). This fact is also consistent with our simulations. As shown in Fig. 2 (d) the vorticity, as predicted after $25 \mathrm{~ns}$ of evolution, is mostly concentrated around the spike's head (so at the bottom of the bubble), yet the re-acceleration phenomenon is believed to be a consequence of the vorticity accumulation at the tip of the bubble. All these elements confirm that the re-acceleration stage has been approached but not yet reached.

We also notice that our MZ width measurements do not match classical buoyancy-drag models as presented in $[13,54,55]$, neither improved ones taking into account time-dependent accelerations $[50,56]$. In every cases, taking our modelled acceleration as an input, the MZ is under-estimated by a factor higher than two. Furthermore, the obtained order for the MZ growth as a function of the foam density is contrary to the experiment: a quicker growth of the $500 \mathrm{mg} \mathrm{cm}^{-3}$ compared to the $20 \mathrm{mg} \mathrm{cm}^{-3}$ is predicted. This can be seen on Fig. 4 (a), where the model, which bear the best results [50], is displayed in dashed line.

The MZ width evolution, however, is not solely due to RTI growth. Material expansion should also be taken into account and may represent a large fraction of the MZ [14]. In contrary, pure, non-HED, RTI develops in the absence of shock waves so there is no interaction with a shock or expansion to consider. Following the procedure described in [51, 52], the background decompression contribution is evaluated by considering the velocity divergence taken from 1D FLASH4 simulations [57]. This leads to values closer to our experimental observations 
(see Fig. 4 (a) plain lines), but it does not reorder the curves as needed. In particular, the $20 \mathrm{mg} \mathrm{cm}^{-3}$ remains underestimated. This could be due to inaccuracy in the foam EOS for this low-density material [58]. However, our results at $A_{t} \simeq 1$ corroborate recent works showing that RTI spikes exhibit a strong dependence on the Atwood number in HED [27, 56]. Spikes could enter in a free-fall regime for any acceleration profile at lower Atwood than predicted by all models. The presence of self-generated magnetic field (Biermann-battery effects) is also not taken into account in the models. Magnetic fields wrap around the RTI spikes [33], laterally confining and sharpening them, enhancing their growth. The actual bubble morphology, as observed in Fig. 1, with a thin wedge like extremity at late time (b-c), is consistent with such interpretation. We can also note that this structure is more prominent in the lighter foam case. Investigating further low density foams $\left(A_{t} \simeq 1\right)$ in the deep nonlinear stage is relevant for ICF. A higher than expected RTI growth was also observed and remains unexplained in highly nonlinear ablative RTI experiments $[37,38]$.

\section{CONCLUSION}

In summary, we report on a parametric study of the RTI in decelerating phase. We performed experiments on the LULI2000 high power laser facility using a direct drive approach to put into motion the modulated rear side of a solid target. The expansion of this target into a foam, used as a deceleration medium, is RT unstable. In these HED experiments, we changed the foam density resulting in both a variation of initial Atwood number across the interface and a variation interface deceleration. Both effects have an opposite contribution to the RTI growth, leading to a competition between them. By analogy with SNR case and by proceeding to some physical consideration, we developed an analytic model to describe the interface motion. Strikingly, this simple model reproduces our experimental data reasonably well with regards to the motion of this interface. Concerning the RTI growth, we note that the perturbation has a faster growth with a low density foam, so in the case of high Atwood number and low deceleration. However, given the acceleration obtained by derivation of our model, the obtained growths cannot be explained using classical RTI models such as the buoyancy-drag model. We attribute this fact to the specificity of the HED physical domain. Mainly, the pressure gradient, due the proximity of the shock and expansion of the plasma, modifies the classical RTI growth, in particular by obstructing the spike growth. We ascertain the role of this gradient, using FLASH4 simulations, and add its effect to the classical RTI growth model. The result is closer to the experiment, but still the agreement is not perfect. Other phenomena, such as the effect of the magnetic field or the re-acceleration, might also play a role and should be considered in future work.

We believe that scaling this platform on MJ scale lasers facilities [53] in association with high resolution x-ray imaging diagnostics [36] could provide an accurate tool to study the heretofore inaccessible role of vorticity in late time RTI growth [21].

\section{ACKNOWLEDGMENTS}

The authors thank the engineering, target fabrication, and operations teams of the LULI laser facility. Target production was realized by P. Ariyathilaka and C. Spindloe from Scitech. This work was supported by the Agence Nationale de la Recherche in the framework of the ANR project TURBOHEDP (ANR-15-CE30-0011).

\section{Appendix A: Errors and Uncertainties}

In this article, error bars and uncertainties appear in several place, but they do not always hold the same meaning. We should distinguish between three kind of error bars. The first one is linked to the measure of different position (spike, bubbles) on the radiographs and simulations. The figures 2 (a) and 3 (a) possess this kind of error bar. The second kind corresponds to the error of the value calculated from the data of the first category. This error is directly derived from the first one and can be seen as the propagation of the previous error. This mainly concerns the figure 4 (a). Finally the error, which are linked to the model and the regression, make the third kind.

\section{Error on the interface position}

The error in the measure position on the experimental x-ray radiographs has several origin. First the x-ray radiograph have some limitation in resolution. This is partly due to the photo-cascade inside the image plate, which broaden the zone being illuminated. Another point to consider is the geometry of the radiography system, a point projection scheme. Due to the shadow and penumbra geometrical property the resolution is limited to the size of the source, $\sim 25 \mu \mathrm{m}$. The temporal deterioration of the resolution due to motion blurring is here negligible compared to the other phenomenon as the exposure time is of the order of $10 \mathrm{ps}$ for a shock velocity lower than $30 \mathrm{um} \mathrm{ns}^{-1}$.

To the previous effects, which are intrinsic to the radiography technique employ, we should add the possible spatial variation of the lighting. These variations are due to phenomena of self-shadowing of the back-lighter, and other variation that may affect the x-ray source (interaction of a titanium wire and a picosecond laser). This leads to spatial non-uniformity is the lighting, which may be a source of error for our measure in some specific cases, 


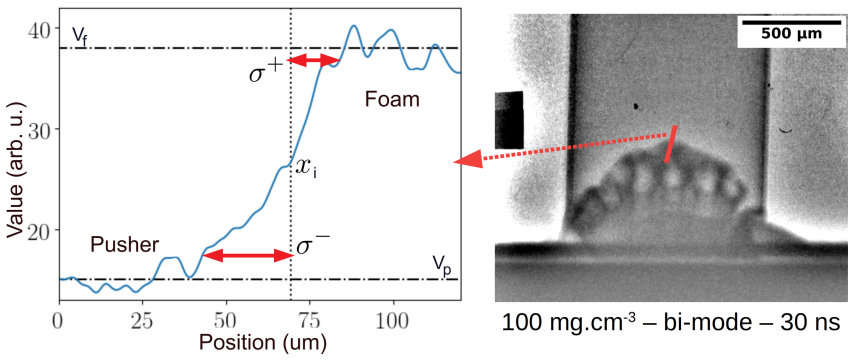

FIG. 5. Position of the spike and asymmetric error bars. A line-out performed is performed on a x-ray radiograph of a $100 / \mathrm{mg} / \mathrm{cm}^{3}$ bi-mode target, $30 \mathrm{~ns}$ after laser shot. This line-out centered on the spike position shows an asymmetry on its intensity profile. This asymmetry is used to define the error bars.

where an abrupt variation coincide with the interface position.

Finally the diffusion of the plasma at the interface between pusher and foam lead to the apparition of absorption gradients. As a result, there is no sharp interface at late time.

Two approaches, which bear sensibly the same results, were employed to measure the interface position. The simplest one, described in [40], consists on finding the interface by eye. The appreciation of each image in its entirety allow surprisingly good results for this method.

The second method consist on performing line-out on the spike and bubble axis (see Fig. 5). On these line-out the signal above the pusher $\left(V_{p}\right)$ is different than the one above the foam $\left(V_{f}>V_{p}\right)$. The interface correspond to the transition between both. We should mention here that this transition is not always symmetric. In such a case, we define the position of the interface $\left(x_{i}\right)$ at the mid-value $\left(V\left(x_{i}\right)=\left(V_{p}+V_{f}\right) / 2\right)$ and we measure both positive $\left(\sigma^{+}=x_{+}-x_{i}\right.$, with $\left.V\left(x_{+}\right)=0.9 V_{f}+0.1 V_{p}\right)$ and negative $\left(\sigma^{-}=x_{i}-x_{-}\right.$, with $V\left(x_{-}\right)=0.1 V_{f}+$ $\left.0.9 V_{p}\right)$ error bars. These results in asymmetric errors. Some other methods exist $[32,59]$ but they could not be employed in the present experiments.

\section{Mixing zone - error composition}

To obtain the mixing zone width, we subtracted the bubble position to the position of the spike. Since both possess asymmetric error, the error on the mixing zone is a composition of both error [60]. Here we define the error

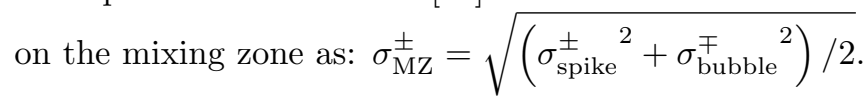
This formula is leads to an over-estimation of the error.

\section{Fitting and error}

The last type of error previously mentioned are the uncertainties related to the model and the value of its fitting parameters. These parameters are obtained by fitting data with asymmetrical errors. To do so we supposed that the asymmetric error bars correspond to two half-normal deviation. We randomly takes a value for each data point using such probability distribution and proceed to the fitting of the data using a least square method. We repeat this process 2000 times and obtained a distribution of possible values for each parameters. This allow us to define the value of each parameter and their asymmetric error bars. In this paper we only keep the higher value for their error making it symmetric.

\section{Appendix B: Buoyancy-drag model application}

One of the unexpected results of this article is the non-compliance of the experiment to the buoyancy-drag model or as shown on fig. 4 (a) to an improve version of it. This came as a surprise since these models can be used to describe the global growth of the RTI, even though they are more often used to describe late time evolution of the instability.

\section{Model and method}

The model evolution displayed on fig. 4 (a) in dashed lines is a direct application of the improve buoyancy-drag model with time dependent acceleration directly taken from [50]. In this model, the following equation is solved:

$$
\ddot{\theta}_{L}-g(t) k_{L} A_{L} \theta_{L}=0
$$

with $\theta_{L} \equiv e^{k_{L}\left(\eta-\eta_{0}\right)}, k_{L} \equiv\left(c(1+c)\left(1+A_{t}\right) k\right) /(2+2 c+$ $\left.2 c A_{t}-2 A_{t}\right), A_{L} \equiv 2 A_{t} /\left(1+c+c A_{t}-A_{t}\right)$. Here, $\eta$ is the the amplitude of the modulation, $\eta_{0}=\eta(t=0)$ its initial value, $g$ is the opposite of the acceleration of the interface obtained through our model $(-\ddot{x}), k$ is the wave-vector of the perturbation, and $A_{t}$ the Atwood number. $c$ is a parameter, which depends on the dimension of the problem being equal to 2 in $2 \mathrm{D}$ and 1 in 3D. In fig. 4 (a), $c$ is taken equal to 1 , but there is no significant difference when using its other value. The definition of $g$ as the opposite of the acceleration is taken in order to be consistent with [50]'s definition (positive Atwood and an acceleration of the interface, $g$, defined positive when pointing toward the high density medium).

To solve this model, a fourth order Runge-Kutta algorithm was implemented. The initial parameters were taken to correspond to the experiment. The initial Atwood number was used as is, not taking into account the compression due to the shock and the following expansion. The same was done for the interface perturbation, which was initialized with a $10 \mu \mathrm{m}$ amplitude and no initial velocity. On that topic, we should report the use of others initial parameters, whose results are not displayed in this article. Indeed, we also tried to use for the initial amplitude and velocity the value obtained in our simulations after shock breakthrough, as well as other value 
in between. The addition of a initial velocity allows us to reproduce the initial RTI growth up to approximately $10 \mathrm{~ns}$, when fine tuned. However, the model gives result similar to those displayed on fig. 4 (a) for later time. The initial velocity just adds a slight concavity at the beginning of the curve before it transition to its first convexity.

Classical buoyancy-drag model were also tried without more success. They were solved using a fourth order Runge-Kutta algorithm and used the same initial parameters as reported above. The only exception is the acceleration which should be fixed for this classical models and was thus taken to its mean value (we also tried a time dependent one).

\section{1D expansion}

As report in the article, one of the downfalls of the previous model is its inability to take into account HED phenomena, such as the pressure gradient resulting from the presence of a shock and the expansion of the material. To take into account such expansion, we followed the method described in $[51,52,57]$. This method follow several steps.

First at least four simulations should be performed. Two are 2D simulations, one of which should reproduce the experimental results with the modulated target, the other one uses a flat target. The simulation with a modulated target is both used to fine tune the laser energy used in simulations, and to obtain a better time discretization for the temporal position of both spike and bubbles. The other simulations are 1D simulations. Since the modulation of the interface cannot be reproduce in $1 \mathrm{D}$, three value for the initial thickness of the pusher can be chosen: the spike $(30 \mu \mathrm{m})$, the bubble $(50 \mu \mathrm{m})$, or the interface in between $(40 \mu \mathrm{m})$. Since the simulation code will give slightly different results in 1 and $2 \mathrm{D}$, we fine tune the laser energy used in $1 \mathrm{D}$ using the $2 \mathrm{D}$ simulation of the flat target and the $1 \mathrm{D}$ with a $40 \mu \mathrm{m}$ pusher. Finally, the $1 \mathrm{D}$ simulation using a $50 \mu \mathrm{m}$ pusher is performed. The $30 \mu \mathrm{m}$ pusher simulation can also be performed at that point, but it holds less value in our case, since the RTI growth comes mainly from the bubbles.

The second step consists on extracting the fluid velocities at the right position from the $1 \mathrm{D}$ simulations. By using 1D simulations the fluid velocity depends only on the expansion of the material and it does not contained the RTI contribution, contrary to $2 \mathrm{D}$ simulations. The "right" position is the position of the bubbles, spikes, interface taken from the $2 \mathrm{D}$ simulations and reported on their respective 1D simulations. In other words, we extract the fluid velocity from the $1 \mathrm{D}$ simulations at the position, where an element characterizing the RTI is expected to be with more dimensions. Since the time steps might not match exactly between $1 \mathrm{D}$ and $2 \mathrm{D}$, and since the spatial resolution will also differ due to the AMR, we proceed to two consecutive cubic interpolations. The first one on the temporal evolution of the RTI elements as seen in $2 \mathrm{D}$, so we can find its position for each time step of the corresponding 1D simulation. The second one is on the spatial velocity variation of the $1 \mathrm{D}$ simulations, to obtain its value at the right position.

From the previously obtained velocities, an expansion velocity is calculated. It consist on the $1 \mathrm{D}$ velocity difference between interface and bubble or interface ans spike. By integrating this expansion velocity, time dependent spatial expansions, $E(t)$ are obtained both in the bubble and spike direction.

$$
E_{\text {bubble }}(t)=\int_{0}^{t}\left(v_{\text {interface }}\left(t^{\prime}\right)-v_{\text {bubble }}\left(t^{\prime}\right)\right) d t^{\prime}
$$

This spatial expansion is simply add to the results of the buoyancy-drag model. Thus, the resulting RTI amplitude, $\tilde{\eta}$, is equal to $\tilde{\eta}(t)=\eta(t)+E(t)$ (reusing previous notation).

\section{Appendix C: Physical parameters}

Thereafter some physical parameters that characterized this experiment are displayed in a table. They were obtained through our 2D simulations. 


\begin{tabular}{|c|c|c|c|}
\hline Parameter & Formula & $20 \mathrm{mg} \mathrm{cm}^{-3}$ & $500 \mathrm{mg} \mathrm{cm}^{-3}$ \\
\hline Shocked foam density $\left(\rho, \mathrm{mg} \mathrm{cm}^{-3}\right)$ & Simulated & 0.4 to 0.6 & 0.8 to 8.7 \\
\hline Temperature $\left(T_{e}=T_{i}, \mathrm{eV}\right)$ & Simulated & 5.3 & 4.9 \\
\hline Pressure (Mbar) & Simulated & 0.05 & 0.11 \\
\hline Ionization $(Z)$ & Thomas-Fermi model & 1.16 & 1.29 \\
\hline Ion density $\left(n_{i}, 10^{10} \mu^{-3}\right)$ & $\rho / m_{i}$ & 3.1 & 6.2 \\
\hline Electron density $\left(n_{e}, 10^{10} \mu^{-3}\right)$ & $n_{i} Z$ & 3.6 & 8.0 \\
\hline Coulomb logarithm $(\ln \Lambda)$ & {$[61]$} & 2.7 & 2.2 \\
\hline Ion thermal collision frequency $\left(\tau_{i}, 10^{5} \mathrm{~ns}^{-1}\right)$ & $4.8 \cdot 10^{-8} Z^{4} n_{i} \ln \Lambda /\left(\left(m_{i} / \text { u.a. }\right)^{0.5} T_{i}^{1.5}\right)$ & 2.1 & 5.6 \\
\hline Ion thermal velocity $\left(v_{i}, \mu \mathrm{mns} \mathrm{n}^{-1}\right)$ & $9.79 \cdot 10^{5}\left(T_{i} /\left(m_{i} / \text { u.a. }\right)\right)^{0.5}$ & 7.9 & 7.6 \\
\hline Ion collisional length $\left(10^{-5} \mu \mathrm{m}\right)$ & $v_{i} / \tau_{i}$ & 3.8 & 1.4 \\
\hline Electron thermal collision frequency $\left(\tau_{e}, 10^{7} \mathrm{~ns}^{-1}\right)$ & $2.91 \cdot 10^{-6} n_{e} \ln \Lambda T_{e}^{-1.5}$ & 2.3 & 4.6 \\
\hline Electron thermal velocity $\left(v_{e}, 10^{2} \mathrm{~mm} \mathrm{~ns}^{-1}\right)$ & $4.19 \cdot 10^{7} T_{e}^{0.5}$ & 9.7 & 9.3 \\
\hline Electron collisional length $\left(10^{-5} \mu \mathrm{m}\right)$ & $v_{e} / \tau_{e}$ & 4.2 & 2.0 \\
\hline Radiative cooling time $\left(10^{4} \mathrm{~ns}\right)$ & {$[62]$ eq. $(22)$} & 9.9 & 2.5 \\
\hline
\end{tabular}

TABLE I. Different physical parameters obtained from the FLASH simulations 30 ns after laser shot. The shock foam density shows the variation of density from the shock to the place near the expanding pusher. $u a$ is the atomic mass unit.

[1] L. Rayleigh, Investigation of the character of the equilibrium of an incompressible heavy fluid of variable density, Proceeding of the London Mathematical Society 14, 170 (1883).

[2] G. I. Taylor, The instability of liquid surfaces when accelerated in a direction perpendicular to their planes, Proceeding of the Royal Society of London A201, 192 (1950).

[3] K. Sasaki, N. Suzuki, D. Akamatsu, and H. Saito, Rayleigh-Taylor instability and mushroom-pattern formation in a two-component bose-einstein condensate, Physical Review A 80, 063611 (2009).

[4] R. Chevalier and R. Klein, On the Rayleigh-Taylor instability in stellar explosions, The Astrophysical Journal 219, 994 (1978).

[5] J. B. Bell, M. S. Day, C. A. Rendleman, S. E. Woosley, and M. Zingale, Direct numerical simulations of type ia supernovae flames. II. the Rayleigh-Taylor instability, The Astrophysical Journal 608, 883 (2004).

[6] U. D'Ortona and N. Thomas, Self-induced RayleighTaylor instability in segregating dry granular flows, Phys. Rev. Lett. 124, 178001 (2020).

[7] A. Hillier, The magnetic Rayleigh-Taylor instability in solar prominences, Reviews of Modern Plasma Physics 2, 1 (2018).

[8] W. Gorczyk, B. Hobbs, and T. Gerya, Initiation of Rayleigh-Taylor instabilities in intra-cratonic settings, Tectonophysics 514, 146 (2012).

[9] C. P. Conrad and P. Molnar, The growth of RayleighTaylor-type instabilities in the lithosphere for various rheological and density structures, Geophys. J. Int. 129, 95 (1997).

[10] R. D. Petrasso, Rayleigh's challenge endures, Nature 367, 217 (1994).

[11] A. Casner, C. Mailliet, G. Rigon, S. Khan, D. Martinez, B. Albertazzi, T. Michel, T. Sano, Y. Sakawa, P. Tzeferacos, D. Lamb, S. Liberatore, N. Izumi, D. Kalantar, P. D. Nicola, J. D. Nicola, E. L. Bel, I. Igumenshchev, V. Tikhonchuk, B. Remington, J. Ballet, E. Falize, L. Masse, V. Smalyuk, and M. Koenig, From
ICF to laboratory astrophysics: ablative and classical Rayleigh-Taylor instability experiments in turbulent-like regimes, Nuclear Fusion 59, 032002 (2018).

[12] Y. Zhou, Rayleigh-Taylor and Richtmyer-Meshkov instability induced flow, turbulence, and mixing. I, Physics Reports 720-722, 1 (2017).

[13] Y. Zhou, Rayleigh-Taylor and Richtmyer-Meshkov instability induced flow, turbulence, and mixing. II, Physics Reports 723-725, 1 (2017).

[14] O. Schilling, Progress on Understanding Rayleigh-Taylor Flow and Mixing Using Synergy Between Simulation, Modeling, and Experiment, Journal of Fluids Engineering 142, 120802 (2020).

[15] P. Ramaprabhu, G. Dimonte, Y.-N. Young, A. C. Calder, and B. Fryxell, Limits of the potential flow approach to the single-mode Rayleigh-Taylor problem, Phys. Rev. E 74, 066308 (2006).

[16] A. Hillier, Self-similar solutions of asymmetric RayleighTaylor mixing, Physics of Fluids 32, 015103 (2020), https://doi.org/10.1063/1.5130893.

[17] D. Layzer, On the Instability of Superposed Fluids in a Gravitational Field., The Astrophysical Journal 122, 1 (1955).

[18] V. N. Goncharov, Analytical model of nonlinear, singlemode, classical Rayleigh-Taylor instability at arbitrary atwood numbers, Phys. Rev. Lett. 88, 134502 (2002).

[19] P. Ramaprabhu, G. Dimonte, P. Woodward, C. Fryer, G. Rockefeller, K. Muthuraman, P.-H. Lin, and J. Jayaraj, The late-time dynamics of the single-mode Rayleigh-Taylor instability, Physics of Fluids 24, 074107 (2012).

[20] T. Wei and D. Livescu, Late-time quadratic growth in single-mode Rayleigh-Taylor instability, Phys. Rev. E 86, 046405 (2012).

[21] X. Bian, H. Aluie, D. Zhao, H. Zhang, and D. Livescu, Revisiting the late-time growth of single-mode RayleighTaylor instability and the role of vorticity, Physica D: Nonlinear Phenomena 403, 132250 (2020).

[22] R. Betti and J. Sanz, Bubble acceleration in the ablative Rayleigh-Taylor instability, Phys. Rev. Lett. 97, 205002 
(2006).

[23] D. S. Clark and M. Tabak, Nonlinear Rayleigh-Taylor growth in converging geometry, Phys. Rev. E 71, 055302 (2005).

[24] L. Pickworth, B. Hammel, V. Smalyuk, H. Robey, R. Tommasini, L. Benedetti, L. Berzak Hopkins, D. Bradley, M. Dayton, S. Felker, et al., Development of new platforms for hydrodynamic instability and asymmetry measurements in deceleration phase of indirectly driven implosions on NIF, Physics of Plasmas 25, 082705 (2018).

[25] J. P. Sauppe, S. Palaniyappan, B. J. Tobias, J. L. Kline, K. A. Flippo, O. L. Landen, D. Shvarts, S. H. Batha, P. A. Bradley, E. N. Loomis, N. N. Vazirani, C. F. Kawaguchi, L. Kot, D. W. Schmidt, T. H. Day, A. B. Zylstra, and E. Malka, Demonstration of scale-invariant Rayleigh-Taylor instability growth in laser-driven cylindrical implosion experiments, Phys. Rev. Lett. 124, 185003 (2020).

[26] J. W. Jacobs and I. Catton, Three-dimensional RayleighTaylor instability Part 2. Experiment, Journal of Fluid Mechanics 187, 353 (1988).

[27] R. Morgan, W. Cabot, J. Greenough, and J. W. Jacobs, Rarefaction-driven Rayleigh-Taylor instability. part 2. experiments and simulations in the nonlinear regime, Journal of Fluid Mechanics 838 (2018).

[28] R. Morgan and J. Jacobs, Experiments and simulations on the turbulent, rarefaction wave driven RayleighTaylor instability, Journal of Fluids Engineering (2020).

[29] Z. Huang, A. De Luca, T. J. Atherton, M. Bird, C. Rosenblatt, and P. Carlès, Rayleigh-Taylor instability experiments with precise and arbitrary control of the initial interface shape, Phys. Rev. Lett. 99, 204502 (2007).

[30] S. S. Orlov, S. I. Abarzhi, S. B. Oh, G. Barbastathis, and K. R. Sreenivasan, High-performance holographic technologies for fluid-dynamics experiments, Philosophical Transactions of the Royal Society A: Mathematical, Physical and Engineering Sciences 368, 1705 (2010).

[31] B. A. Remington, H.-S. Park, D. T. Casey, R. M. Cavallo, D. S. Clark, C. M. Huntington, C. C. Kuranz, A. R. Miles, S. R. Nagel, K. S. Raman, et al., RayleighTaylor instabilities in high-energy density settings on the National Ignition Facility, Proceedings of the National Academy of Sciences 116, 18233 (2019).

[32] J. D. Bender, O. Schilling, K. S. Raman, R. A. Managan, B. J. Olson, S. R. Copeland, C. L. Ellison, D. J. Erskine, C. M. Huntington, B. E. Morgan, S. R. Nagel, S. T. Prisbrey, B. S. Pudliner, P. A. Sterne, C. E. Wehrenberg, and Y. Zhou, Simulation and flow physics of a shocked and reshocked high-energy-density mixing layer, Journal of Fluid Mechanics 915, A84 (2021).

[33] C. C. Kuranz, R. P. Drake, M. J. Grosskopf, B. Fryxell, A. Budde, J. S. Hansen, A. R. Mile, T. Plewa, N. Heam, and J. Knauer, Spike morphology in blast-wave-driven instability experiments, Physics of Plasmas 17, 052709 (2010).

[34] C. C. Kuranz, H.-S. Park, C. M. Huntington, A. R. Miles, B. A. Remington, T. Plewa, M. R. Trantham, H. F. Robey, D. Shvarts, A. Shimony, K. Raman, S. MacLaren, W. C. Wan, F. W. Doss, J. Kline, K. A. Flippo, G. Malamud, T. A. Handy, S. Prisbrey, C. M. Krauland, S. R. Klein, E. C. Harding, R. Wallace, M. J. Grosskopf, D. C. Marion, D. Kalantar, E. Giraldez, and R. P. Drake, How high energy fluxes may affect Rayleigh-Taylor instability growth in young supernova remnants, Nature Communications 9, 1564 (2018).

[35] G. Rigon, A. Casner, B. Albertazzi, T. Michel, P. Mabey, E. Falize, J. Ballet, L. Van Box Som, S. Pikuz, Y. Sakawa, T. Sano, A. Faenov, T. Pikuz, N. Ozaki, Y. Kuramitsu, M. P. Valdivia, P. Tzeferacos, D. Lamb, and M. Koenig, Rayleigh-Taylor instability experiments on the LULI2000 laser in scaled conditions for young supernova remnants, Phys. Rev. E 100, 021201 (2019).

[36] G. Rigon, B. Albertazzi, T. Pikuz, P. Mabey, V. Bouffetier, N. Ozaki, T. Vinci, F. Barbato, E. Falize, Y. Inubushi, N. Kamimura, K. Katagiri, S. Makarov, M. J.-E. Manuel, K. Miyanishi, S. Pikuz, O. Poujade, K. Sueda, T. Togashi, Y. Umeda, M. Yabashi, T. Yabuuchi, G. Gregori, R. Kodama, A. Casner, and M. Koenig, Micronscale phenomena observed in a turbulent laser-produced plasma, Nature Communications 12, 2679 (2021).

[37] D. A. Martinez, V. A. Smalyuk, J. O. Kane, A. Casner, S. Liberatore, and L. P. Masse, Evidence for a bubble-competition regime in indirectly driven ablative Rayleigh-Taylor instability experiments on the NIF, Phys. Rev. Lett. 114, 215004 (2015).

[38] A. Casner, L. Masse, S. Liberatore, P. Loiseau, P. E. Masson-Laborde, L. Jacquet, D. Martinez, A. S. Moore, R. Seugling, S. Felker, S. W. Haan, B. A. Remington, V. A. Smalyuk, M. Farrell, E. Giraldez, and A. Nikroo, Probing the deep nonlinear stage of the ablative Rayleigh-Taylor instability in indirect drive experiments on the National Ignition Facility, Physics of Plasmas 22, 056302 (2015), https://aip.scitation.org/doi/pdf/10.1063/1.4918356.

[39] E. Brambrink, S. Baton, M. Koenig, R. Yurchak, N. Bidaut, B. Albertazzi, J. Cross, G. Gregori, A. Rigby, E. Falize, A. Pelka, F. Kroll, S. Pikuz, Y. Sakawa, N. Ozaki, C. C. Kuranz, M. Manuel, C. Li, P. Tzeferacos, and D. Lamb, Short-pulse laser-driven x-ray radiography, HPLSE 4, 31 (2016).

[40] N. C. Swisher, C. C. Kuranz, D. Arnett, O. Hurricane, B. A. Remington, R. H. F., and S. I. Abarzhi, Rayleightaylor mixing in supernova experiments, Physics of Plasmas 22, 102707 (2015).

[41] B. Fryxell, K. Olson, P. Ricker, F. X. Timmes, M. Zingale, D. Q. Lamb, P. MacNeice, R. Rosner, J. W. Truran, and H. Tufo, Flash: An adaptive mesh hydrodynamics code for modeling astrophysical thermonuclear flashes, Astrophysical Journal, Supplement 131, 273 (2000).

[42] A. Dubey, A. Almgren, J. Bell, M. Berzins, S. Brandt, G. Bryan, P. Colella, D. Graves, M. Lijewski, F. Löffler, B. O'Shea, E. Schnetter, B. Van Straalen, and K. Weide, A survey of high level frameworks in block-structured adaptive mesh refinement packages, Journal of Parallel and Distributed Computing 74, 3217 (2014).

[43] J. Macfarlane, Ionmix - a code for computing the equation of state and radiative properties of lte and nonlte plasmas, Computer Physics Communications 56, 259 (1989).

[44] J. MacFarlane, I. Golovkin, and P. Woodruff, Helios-cr a 1-d radiation-magnetohydrodynamics code with inline atomic kinetics modeling, Journal of Quantitative Spectroscopy and Radiative Transfer 99, 381 (2006), radiative Properties of Hot Dense Matter.

[45] S. G. Glendinning, J. Bolstad, D. Braun, M. Edwards, W. Hsing, B. Lasinski, H. Louis, A. Miles, J. Moreno, T. Peyser, et al., Effect of shock proximity on Richtmyer- 
Meshkov growth, Physics of Plasmas 10, 1931 (2003).

[46] J. K. Truelove and C. F. McKee, Evolution of Nonradiative Supernova Remnants, The Astrophysical Journal Supplement Series 120, 299 (1999).

[47] X. Tang and R. A. Chevalier, Shock evolution in nonradiative supernova remnants, Monthly Notices of the Royal Astronomical Society 465, 3793 (2017).

[48] J. M. Blondin and D. C. Ellison, Rayleigh-Taylor instabilities in young supernova remnants undergoing efficient particle acceleration, The Astrophysical Journal 560, 244 (2001).

[49] F. Fraschetti, R. Teyssier, J. Ballet, and A. Decourchelle, Simulation of the growth of the $3 \mathrm{~d}$ rayleigh-taylor instability in supernova remnants using an expanding reference frame, Astronomy \& Astrophysics 515, A104 (2010).

[50] K. O. Mikaelian, Nonlinear hydrodynamic interface instabilities driven by time-dependent accelerations, Physical Review E 79, 065303 (2009).

[51] A. R. Miles, Bubble merger model for the nonlinear Rayleigh-Taylor instability driven by a strong blast wave, Physics of Plasmas 11, 5140 (2004), https://doi.org/10.1063/1.1790498.

[52] A. R. Miles, The blast-wave-driven instability as a vehicle for understanding supernova explosion structure, The Astrophysical Journal 696, 498 (2009).

[53] G. Malamud, L. Elgin, T. Handy, C. Huntington, R. Drake, D. Shvarts, A. Shimony, and C. Kuranz, Design of a single-mode Rayleigh-Taylor instability experiment in the highly nonlinear regime, High Energy Density Physics 32, 18 (2019).

[54] G. Dimonte, Spanwise homogeneous buoyancy-drag model for rayleigh-taylor mixing and experimental eval- uation, Physics of Plasmas 7, 2255 (2000).

[55] D. Oron, L. Arazi, D. Kartoon, A. Rikanati, U. Alon, and D. Shvarts, Dimensionality dependence of the RayleighTaylor and Richtmyer-Meshkov instability late-time scaling laws, Physics of Plasmas 8, 2883 (2001).

[56] P. Ramaprabhu, V. Karkhanis, R. Banerjee, H. Varshochi, M. Khan, and A. G. W. Lawrie, Evolution of the single-mode Rayleigh-Taylor instability under the influence of time-dependent accelerations, Phys. Rev. E 93, 013118 (2016).

[57] R. P. Drake, Spike penetration in blast-wave-driven instabilities, The Astrophysical Journal 744, 184 (2011).

[58] K. Nagai, C. S. Musgrave, and W. Nazarov, A review of low density porous materials used in laser plasma experiments, Physics of Plasmas 25, 030501 (2018).

[59] C. Huntington, K. Raman, S. Nagel, S. MacLaren, T. Baumann, J. Bender, S. Prisbrey, L. Simmons, P. Wang, and Y. Zhou, Split radiographic tracer technique to measure the full width of a high energy density mixing layer, High Energy Density Physics 35, 100733 (2020).

[60] R. Barlow, Asymmetric errors (2004), arXiv:physics/0401042 [physics.data-an].

[61] T. White, M. Oliver, P. Mabey, and et al., Supersonic plasma turbulence in the laboratory, Nat Commun 10, 1758 (2019).

[62] D. Ryutov, R. P. Drake, J. Kane, E. Liang, B. A. Remington, and W. M. Wood-Vasey, Similarity criteria for the laboratory simulation of supernova hydrodynamics, ApJ 518, 821 (1999). 